Solange Veloso Viana' Maria Cecilia Costa ${ }^{2}$ Sebastião Loureiro ${ }^{3}$ Iracema Santos Veloso ${ }^{4}$

\section{Ocorrência de sobrepeso e outros fatores de ris- co cardiovascular em trabalhadores atendidos em um ambulatório de nutrição do Centro de Estudos de Saúde do Trabalhador, Salvador-BA}

\author{
Occurrence of overweight and other risk fac- \\ tors among workers attending a nutrition and \\ health surveillance outpatient clinic in the \\ state of Bahia, Brazil
}

\begin{abstract}
Resumo
O estudo objetiva determinar a ocorrência de sobrepeso e suas possíveis associações com o perfil lipídico indesejável e a adiposidade central em trabalhadores atendidos no ambulatório de nutrição do Centro de Estudos de Saúde do Trabalhador (CESAT), em Salvador-Bahia. Buscando produzir informações que possam apoiar o desenvolvimento de ações voltadas para o controle dos riscos ou agravos à saúde, o estudo desenvolveu-se entre 1995 e 2000 como atividade de estágio do Programa de Nutrição e Vigilância à Saúde do Adulto da Universidade Federal da Bahia. Após análise e organização do banco de dados, selecionaram-se 1066 trabalhadores provenientes de uma demanda espontânea a este serviço. Deste universo, definiram-se subgrupos específicos para análise mais detalhada de variáveis relevantes para o estudo, considerando também a disponibilidade dos dados. Assim, foi estimada a Freqüência (F), a Razão de Prevalência (RP) com seus Intervalos de Confiança a 95\% (IC 95\%) e o teste de proporção (t). Os resultados mostram elevadas freqüências de sobrepeso, dislipidemia e hipertensão arterial nestes trabalhadores de ambos os sexos. As mulheres apresentam maior adiposidade central e o índice de Castelli. O cálculo da RP entre sobrepeso, dislipidemia, adiposidade central e RCQ mostrou uma associação estatisticamente significante. O teste de proporção mostrou associação apenas entre sobrepeso e adiposidade central. Os homens apresentaram maior proporção de risco cardiovascular, como hipertensão e baixos valores de colesterol HDL.
\end{abstract}

Palavras-chaves: sobrepeso, fatores de risco, saúde do trabalhador.

\begin{abstract}
The objective of this study is to search for the occurrence of overweight associated to undesirable lipid profile and central obesity on 1,066 workers attending a nutrition and health surveillance clinic in the Workers' Health Study Center in Salvador, Bahia, Brazil. The study was carried out from 1995 to 2000 on subgroups of workers that have fulfilled the criteria of having a minimum set of biochemical and anthropometric data. Data analysis was performed to measure Frequency with Confidence Intervals, Proportion Ratio and Proportion Test. The results have shown that the frequency of hypertension, triglycerides and levels of low HDL-Cholesterol were high among men. Women presented higher frequency of belly circumference, $R C Q$ and Castelli index above the risk limit. Statistical analysis using prevalence ratio demonstrated a significant association of overweight with high serum lipids, belly circumference and the belly to hips ratio (RCQ). Overweight did not show significant difference between men and women, in this study.
\end{abstract}

Keyworks: overweight, risk factors, workers' health. 


\section{Introdução}

No Brasil, a diminuição do risco de morrer por doenças infecciosas e parasitárias vem sendo acompanhada pelo crescimento dos coeficientes de morbi-mortalidade por doenças crônicas não-transmissíveis DCNT. Tais doenças já alcançam no país os primeiros lugares em mortalidade precoce, medida pelos anos produtivos de vida perdidos (ALMEIDA FILHO \& ROUQUAYROL, 1992) e em custos hospitalares.

Assim, no tocante às causas das mortes no país, o fato mais surpreendente referido às últimas décadas tem sido a queda acentuada dos óbitos por doenças infecciosas e a elevada mortalidade por doenças crônicas, a exemplo das cardiovasculares, do diabetes e do câncer (LESSA, 1998; MONTEIRO et al.,1995). De etiologia complexa e de curso prolongado, esses problemas têm sido associados a um conjunto de determinantes, dentre os quais o sobrepeso, as dislipidemias e a adiposidade central.

Nas três últimas décadas, a literatura relata um aumento da prevalência do sobrepeso, superior a 25\% nos Estados Unidos (FLEGAL et al., 1998). Nos países em desenvolvimento, inclusive no Brasil, os estudos apontam o papel importante da mesma e crescente tendência à formação de novos hábitos alimentares. Neste aspecto, um importante dado a considerar referese ao fato de que o aumento do sobrepeso é também uma tendência em todas as regiões do país (MONDINI \& MONTEIRO, 1995). Estudo realizado a partir de inquéritos nutricionais probabilísticos nas três últimas décadas revelou uma evolução ascendente da obesidade na região nordeste, intensificada no período de 1989 a 1997 (MONTEIRO \& CONDE, 1999).

O sobrepeso em graus avançados tem sido mencionado em inúmeros estudos como um dos determinantes mais importantes de várias DCNTs. O sobrepeso isolado revela-se como um fator de risco independente dos demais para a ocorrência de doença isquêmica coronariana e morte súbita, especialmente em homens abaixo de 50 anos, ou seja, na população economicamente ativa (HUBERT et al., 1983).

Assim, o risco de desenvolver diabetes mellitus (DM), dislipidemia e hipertensão arterial sistêmica (HAS) - condições que favorecem o desenvolvimento das doenças cardiovasculares (DCVs) - mostra-se aumentado nos indivíduos com sobrepeso quando comparados àqueles com peso normal (LESSA, 1998).

Bem demonstrada também é a associação da obesidade visceral - causada por vários fatores, dentre os quais idade, genética, lipoproteinolipase e hormônios cortisol e esteróides sexuais - com a HAS. Apesar da associação entre obesidade e hipertensão não estar bem esclarecida, indiscutivelmente a pressão arterial diminui com o emagrecimento. Estudos têm mostrado que modestas reduções de peso (3 a $5 \mathrm{~kg}$ ) diminuem significativamente a pressão, sendo por esta razão a maneira não farmacológica mais efetiva para controlar a hipertensão (SUPLICY, 2000).

Os dados nacionais sobre a epidemiologia do sobrepeso ainda são poucos e mal distribuídos no território nacional. Entretanto, é possível delinear a sua importância na Bahia, exemplificando, com base em estudos recentes, a alta proporção de trabalhadores portadores de tais problemas. Em um estudo realizado com trabalhadores de uma indústria petroquímica baiana, verificou-se que $62 \%$ são portadores de sobrepeso e $47,6 \%$ de hipercolesterolemia (VIANA, 2000). Dados semelhantes são encontrados em um outro estudo, cujos resultados apontam uma proporção de trabalhadores, também do segmento industrial baiano, com $50,6 \%$ de sobrepeso e $49,4 \%$ de colesterol acima do desejável (COSTA, 2000). Uma outra investigação revela o aumento do sobrepeso entre trabalhadores baianos do ramo industrial beneficiários do Programa de Alimentação do Trabalhador - PAT (VELOSO \& SANTANA, 2002). Essa propensão é apontada em um outro estudo que mostra a tendência secular da obesidade de acordo com estratos sociais no nordeste e no sudoeste do Brasil. A partir de inquéritos nutricionais probabilísticos realizados nas três últimas décadas, os autores concluíram como ascendente a evolução da obesidade nas duas regiões no período de 19751979, expresso no aumento mais intenso da obesidade na região nordeste, sobretudo no sexo feminino, e no período 1989-1997 (MONTEIRO \& CONDE, 1999).

Esses dados põem em evidência as transformações nos estilos de vida e no mundo do trabalho, sugerindo a emergência de hábitos que seriam, segundo alguns estudos (EPSTEIN \& HIGGINS, 1992; FON- 
SECA et al., 1999), resultantes do ajuste às novas formas de consumo e aos novos padrões tecnológicos, além dos processos de urbanização e migração.

Diante desse cenário, o estudo do sobrepeso em diferentes graus e dos problemas a ele associados, a exemplo das dislipidemias e da adiposidade central, assume importância particular, uma vez que são múltiplas as evidências dessas associações a várias DCNTs, com destaque especial para a hipertensão arterial, o diabetes e as doenças cardiovasculares. Nesse aspecto, o sobrepeso representa um importante exemplo das interações entre fatores de risco e da possibilidade de confundimento. Assim, fatores de risco comuns à determinação do sobrepeso são também determinantes da hipertensão, do diabetes e das dislipidemias, embora o sobrepeso em si possa determinar, entre outros problemas,

\section{Metodologia}

O estudo, de corte transversal, foi realizado no ambulatório de nutrição do Centro de Estudos de Saúde do Trabalhador (CESAT), na cidade de Salvador, Bahia, e coordenado por professores da Escola de Nutrição da Universidade Federal da Bahia. O ambulatório funciona desde setembro de 1995 atendendo à demanda espontânea ${ }^{5}$ dos trabalhadores com diagnóstico de doença ocupacional. Entre setembro de 1995 e setembro de 2000 , foram selecionados, no universo de 1066 trabalhadores provenientes de uma demanda espontânea, subgrupos de análise tomando como critério de constituição dos grupos a disponibilidade dos dados. Trabalhadores com idade $\geq 20$ anos, de ambos os sexos, com diagnóstico de doença ocupacional foram selecionados e formaram subgrupos para análise das seguintes variáveis:

a) IMC (1066 participantes);

b) Circunferência da Cintura (CC) e Relação Cintura Quadril (RCQ) (767 participantes);

c) Colesterol total (447 participantes);

d) HDL Colesterol (414 participantes);

e) Índice de Castelli (411 participantes);

f) LDL Colesterol (409 participantes);

g) Triglicérides (430 participantes); a hipertensão, o diabetes e outras doenças cardiovasculares (LESSA, 1998).

Tais informações evidenciam a importância do estudo do sobrepeso e outros fatores de riscos para algumas DCNTs, considerando que tais doenças, além de constituírem uma das principais causas de óbito no país, caracterizam-se pela evolução dos graus de incapacidade, morte prematura da população economicamente ativa, além de contribuírem para a elevação dos gastos nos serviços de saúde.

Considerando o papel dos Centros de Estudos de Saúde do Trabalhador (CESAT) na busca dos principais riscos e na organização de informações relacionadas à saúde deste segmento, este estudo teve como objetivo determinar a ocorrência de sobrepeso e sua possível associação com o perfil lipídico indesejável e a adiposidade central de trabalhadores atendidos no ambulatório de Nutrição do CESAT.

h)Dislipidemia (425 participantes);

i) Hipertensão Arterial (HA) (697 participantes);

j) IMC associado a sexo, à dislipidemia, à RCQ, à CC (274 participantes), um subgrupo constituído por trabalhadores que dispunham de todos os dados, inclusive os resultados dos exames bioquímicos.

Embora a solicitação dos exames fosse parte integrante do protocolo de atendimento no ambulatório de nutrição, um número expressivo de trabalhadores deixou de realizá-los por variados motivos, sendo o mais freqüente a dificuldade de acesso aos laboratórios credenciados do SUS.

Dados sobre características pessoais, socioambientais, do trabalho e do estilo de vida foram obtidos por meio de entrevista. Informações sobre peso, altura e circunferências da cintura e quadril foram obtidas por meio de exames antropométricos a partir de técnicas e instrumentos padronizados. Dos prontuários clínicos obtiveram-se informações sobre a presença de DCNT. Dados sobre glicemia, colesterol total, HDL colesterol, LDL colesterol e triglicérides foram obtidos por meio de exames bioquímicos solicitados pelo ambulatório.
${ }^{5}$ Demanda espontânea refere-se à procura do ambulatório de nutrição pelo trabalhador ao ser informado, durante o processo de triagem, dos serviços oferecidos pelo CESAT. 
Para a análise, foi estimada a Freqüência (F), a Razão de Prevalência (RP) com seus Intervalos de Confiança a 95\% (IC 95\%) e teste de proporção (t).

\section{Critérios e definições}

As variáveis selecionadas no estudo como fatores de risco à saúde e seus pontos de corte (MARTINEZ, 1997; SBC, 2001, 2004; WHO, 1997, 2003) estão relacionadas a seguir:

- Sobrepeso em diferentes graus: índice de massa corporal (IMC) $\geq 25 \mathrm{~kg} / \mathrm{m}^{2}$ de superfície corporal;

- Circunferência da Cintura Natural (CC): valores $\geq 102 \mathrm{~cm}$ em homens e $\geq 88 \mathrm{~cm}$ em mulheres, independentemente da presença de sobrepeso generalizado;

- Razão Cintura Quadril (RCQ): circunferência da cintura natural/circunferência do quadril $>1,0$ para homens e > 0,85 para mulheres;

- Colesterol Total (CT): valores $\geq 200$ $\mathrm{mg} / \mathrm{dL}$;

\section{Resultados}

Os resultados gerais descritos na Tabela 1 mostram alta freqüência de sobrepeso,
- HDL Colesterol (HDL-c): valores < 40 $\mathrm{mg} / \mathrm{dL}$;

- LDL Colesterol (LDL-c): valores $\geq 130$ $\mathrm{mg} / \mathrm{dL}$;

- Triglicérides (Tg): valores $\geq 150 \mathrm{mg} /$ $\mathrm{dL}$

- Índice de Castelli: razão entre colesterol total e HDL-c com valores > 5,1 para homens e $>4,4$ para mulheres;

- Dislipidemia: pelo menos uma das lípidas (CT, HDL-C; LDL-c e Tg) alterada;

- Pressão Arterial Sistólica: valores $\geq$ $140 \mathrm{mmHg}$;

- Pressão Arterial Diastólica: valores $\geq$ $90 \mathrm{mmHg}$.

Para a análise, foram calculadas as freqüências (F) das variáveis com seus IC a 95\%, RP do sobrepeso, da dislipidemia e da adiposidade central (CC e RCQ) e foi realizado teste estatístico para evidenciar diferença na proporção do sobrepeso entre os sexos entre os trabalhadores com dislipidemia e com adiposidade central.

Tabela 1 Freqüência das variáveis selecionadas para o estudo, por sexo, do ambulatório de nutrição do CESAT, set/1995 a set/2000, Salvador-BA.

\begin{tabular}{|c|c|c|c|c|c|c|}
\hline \multirow{2}{*}{ Variáveis } & \multicolumn{3}{|c|}{ Masculino } & \multicolumn{3}{|c|}{ Feminino } \\
\hline & $n$ & $F \%$ & IC $95 \%$ & $n$ & $F \%$ & IC 95\% \\
\hline \multicolumn{7}{|l|}{$\mathrm{IMC}\left(\mathrm{Kg} / \mathrm{m}^{2}\right)$} \\
\hline$<25$ & 218 & 44,9 & $(41,9-47,9)$ & 257 & 44,3 & $(41,3-47,3)$ \\
\hline$\geq 25$ & 268 & 55,1 & $(52,1-58,1)$ & 323 & 55,7 & $(52,7-58,7)$ \\
\hline \multicolumn{7}{|l|}{ CC } \\
\hline sem risco & 251 & 86,2 & $(83,8-88,6)$ & 312 & 65,6 & $(62,2-69)$ \\
\hline com risco & 40 & 13,8 & $(11,4-16,2)$ & 164 & 34,4 & $(31-37,8)$ \\
\hline \multicolumn{7}{|l|}{ RCQ } \\
\hline desejável & 283 & 97,2 & $(96-98,4)$ & 322 & 67,6 & $(64,3-70,9)$ \\
\hline indesejável & 8 & 2,8 & $(1,6-4)$ & 154 & 32,4 & $(29,1-35,7)$ \\
\hline \multicolumn{7}{|l|}{ Hipercolesterolemia } \\
\hline não & 76 & 49,3 & $(45,1-54,3)$ & 149 & 50,9 & $(46,3-55,5)$ \\
\hline $\operatorname{sim}$ & 78 & 50,7 & $(45,7-54,9)$ & 144 & 49,1 & $(44,5-53,7)$ \\
\hline
\end{tabular}


(...) Tabela 1 Freqüência das variáveis selecionadas para o estudo, por sexo, do ambulatório de nutrição do CESAT, set/1995 a set/2000, Salvador-BA.

\begin{tabular}{|c|c|c|c|c|c|c|}
\hline \multirow{2}{*}{ Variáveis } & \multicolumn{3}{|c|}{ Masculino } & \multicolumn{3}{|c|}{ Feminino } \\
\hline & $n$ & $F \%$ & IC 95\% & $n$ & $F \%$ & IC 95\% \\
\hline \multicolumn{7}{|l|}{ HDL-c baixo } \\
\hline não & 99 & 70,7 & $(66,8-75,6)$ & 229 & 83,6 & $(79,9-87,1)$ \\
\hline $\operatorname{sim}$ & 41 & 29,3 & $(24,4-33,2)$ & 45 & 16,4 & $(12,9-20,1)$ \\
\hline \multicolumn{7}{|l|}{ Índice de Castelli } \\
\hline desejável & 99 & 71,2 & $(67,3-76,1)$ & 176 & 64,7 & $(60,1-69,3)$ \\
\hline indesejável & 40 & 28,8 & $(23,9-32,7)$ & 96 & 35,3 & $(30,7-39,9)$ \\
\hline \multicolumn{7}{|l|}{ LDL-c indesejável } \\
\hline não & 76 & 54,7 & $(50,3-59,9)$ & 157 & 58,1 & $(53,3-62,9)$ \\
\hline $\operatorname{sim}$ & 63 & 45,3 & $(40,1-49,7)$ & 113 & 41,9 & $(37,1-46,7)$ \\
\hline \multicolumn{7}{|l|}{ Hipertrigliceridemia } \\
\hline não & 101 & 68,2 & $(64,3-73,1)$ & 220 & 78 & $(74-81,8)$ \\
\hline $\operatorname{sim}$ & 47 & 31,8 & $(26,9-35,7)$ & 62 & 22 & $(18,2-26)$ \\
\hline \multicolumn{7}{|l|}{ Dislipidemia } \\
\hline não & 33 & 22,8 & $(18,9-26,9)$ & 93 & 33,2 & $(28,7-37,7)$ \\
\hline $\operatorname{sim}$ & 112 & 77,2 & $(73,1-81,1)$ & 187 & 66,8 & $(62,3-71,3)$ \\
\hline \multicolumn{7}{|l|}{ Hipertensão arterial } \\
\hline não & 163 & 54,3 & $(51-58,4)$ & 257 & 64,7 & $(60,5-67,7)$ \\
\hline $\operatorname{sim}$ & 137 & 45,7 & $(41,6-49)$ & 140 & 35,3 & $(32,3-39,5)$ \\
\hline
\end{tabular}

Os resultados envolvendo o subgrupo de 274 trabalhadores analisados são mostrados na Tabela 2. Observa-se que o grupo de indivíduos do sexo masculino apresentou maior proporção de sobrepeso do que o grupo do sexo feminino, embora essa diferença não seja estatisticamente significante $(R P=1,10, t=0,36)$. Foi possível demonstrar uma associação estatisticamente significante com sobrepeso apenas no grupo que apresentou valores de RCQ
$(\mathrm{RP}=1,68, \mathrm{t}=2,38)$ e CC acima do limite desejável $(\mathrm{RP}=2,11$ e $\mathrm{t}=3,61)$. Por outro lado, a associação entre sobrepeso e adiposidade central revela-se mais elevada entre as mulheres, o que sugere um possível aumento de risco para complicações metabólicas. A associação entre sobrepeso e dislipidemia mostrou-se significante apenas na análise da razão de proporção $(\mathrm{RP}=1,47 \mathrm{e}$ $t=0,66)$.

Tabela 2 Freqüências, razões de prevalência e teste de proporção das variáveis selecionadas no estudo do subgrupo dos trabalhadores atendidos no ambulatório de nutrição do CESAT entre set/1995 e set/2000, Salvador-BA.

\begin{tabular}{|c|c|c|c|c|c|c|c|c|}
\hline Variáveis & $n$ & Eutrofia & IC 95\% & Sobrepeso & IC 95\% & $R P$ & IC 95\% & $t$ \\
\hline \multicolumn{9}{|l|}{ Sexo } \\
\hline masculino & 92 & 32,6 & $(27-38,2)$ & 67,4 & $(61,8-73)$ & & & \\
\hline feminino & 182 & 39 & $(33,2-44,8)$ & 61 & $(55,2-66,8)$ & & & \\
\hline \multicolumn{9}{|l|}{ Dislipidemia } \\
\hline $\operatorname{sim}$ & 192 & 30,2 & $(24,8-365,6)$ & 69,8 & $(64,4-75,2)$ & & & \\
\hline não & 82 & 52,4 & $(46,5-58,3)$ & 47,6 & $(41,7-53,5)$ & & & \\
\hline
\end{tabular}


(...) Tabela 2 Freqüências, razões de prevalência e teste de proporção das variáveis selecionadas no estudo do subgrupo dos trabalhadores atendidos no ambulatório de nutrição do CESAT entre set/1995 e set/2000, Salvador-BA.

\begin{tabular}{|c|c|c|c|c|c|c|c|c|c|}
\hline & Variáveis & $n$ & Eutrofia & IC 95\% & Sobrepeso & IC 95\% & $R P$ & IC 95\% & $t$ \\
\hline \multicolumn{10}{|c|}{ RCQ } \\
\hline & indesejável & 67 & 9 & $(5,6-12,4)$ & 91 & $(87,6-94,4)$ & & & \\
\hline & desejável & 207 & 45,9 & $(40-51,8)$ & 54,1 & $(48,2-60)$ & & & \\
\hline \multicolumn{10}{|c|}{ CC } \\
\hline & com risco & 86 & 1,2 & $(-0,1-2,5)$ & 98,8 & $(97,5-100,1)$ & & & \\
\hline & sem risco & 188 & 53,2 & $(47,3-59,1)$ & 46,8 & $(40,9-52,7)$ & & & \\
\hline & & & & & & & 2,11 & $(1,81-2,46)$ & 3,61 \\
\hline
\end{tabular}

\section{Discussão}

A seleção de amostra não probabilística para o presente estudo limita uma possível estimativa para a população dos parâmetros encontrados. Entretanto, não é este o objetivo do presente estudo, que busca verificar associações entre indicadores antropométricos e de risco cardiovascular em um subgrupo restrito de trabalhadores que procuraram um ambulatório do CESAT. A demonstração de que a associação entre sobrepeso e indicadores de risco cardiovascular está presente entre os trabalhadores pode orientar ações preventivas e de promoção de saúde mais específicas para este grupo estratégico.

A alta freqüência de sobrepeso entre homens e mulheres atendidos no ambulatório de nutrição do CESAT foi confirmada. Esse dado corrobora os estudos que apontam a ausência de diferenças significativas entre os sexos no que se refere à alta prevalência de sobrepeso na população adulta nordestina. Confirma ainda a tendência de aumento de sobrepeso na região nordeste, em ambos os sexos, observada a partir de inquéritos nutricionais probabilísticos realizados nas três últimas décadas (MONTEIRO \& CONDE, 1994).

Embora não estatisticamente significante, o aumento da freqüência de sobrepeso na população masculina do subgrupo que dispunha de todos os dados do estudo mostra-se coerente com os achados que apontam um maior crescimento de obesidade entre os homens nordestinos quando comparados às mulheres da região $(\mathrm{MON}$ TEIRO \& CONDE, 1994).

Também compatível com esses achados é a alta freqüência de perfil lipídico indesejável apresentado por ambos os se- xos nos subgrupos estudados. Esses dados confirmam a estreita relação já descrita pela literatura entre sobrepeso e dislipidemias. Nestes subgrupos, a associação entre altas taxas de dislipidemia e a presença de sobrepeso demonstra não só uma relação entre ambos como uma condição que potencialmente favorece o desenvolvimento de doenças cardiovasculares (CARNEIRO et al., 2003).

As lípidas observadas de forma isolada ou em conjunto nos subgrupos estudados apresentam freqüências elevadas. Tais lípidas, consideradas fatores de riscos tradicionais, têm o potencial de isoladamente induzir a doenças cardiovasculares (SOCESP, 2000).

Ambos os sexos apresentaram, no estudo, valores acima do desejável no que se refere ao Índice de Castelli, embora as mulheres tenham apresentado freqüência mais alta. Esse dado revela uma maior exposição do sexo feminino a doenças cardiovasculares e é compatível com os resultados de um estudo de corte transversal realizado em adultos com idade $\geq 20$ anos em Salvador-BA no ano de 2004 (LESSA et al., 2004).

Embora a hipertensão arterial seja relacionada com a distribuição central da adiposidade corporal (CARNEIRO et al., 2003), no estudo verifica-se maior risco de HA entre os homens, apesar das mulheres apresentarem maior Circunferência Central. Uma possível explicação para este resultado pode ser atribuída aos limites do banco de dados utilizado para o estudo. As informações nele contidas mostraram-se insuficientes para o controle de outros fatores de risco para hipertensão, como histó- 
ria familiar da doença, consumo de álcool, ingestão de sal, atividade física, entre outros (SBH, 2002; SBC, 2004; WHO, 2003). Apesar da limitação dos dados, o subgrupo estudado revela que indivíduos com adiposidade central, independentemente do critério aplicado para a identificação, apresentam associação igualmente elevada com sobrepeso. Esse achado mostra-se particularmente interessante, considerando que estudos mais recentes têm mostrado a medida isolada da CC como suficiente para estabelecer risco de co-morbidades associadas ao sobrepeso, em diferentes graus, com risco substancialmente aumentado para dislipidemias, diabetes e doenças cardiovasculares (HAN et al., 1995; HALPERN \& MANCINI, 2000; KAC et al. 2001; SBC, 2001).

Os resultados deste estudo são semelhantes a outras investigações que mostram o aumento da prevalência de sobrepeso em seus diversos graus, na região nordeste, e a sua importância no desenvolvimento de

\section{Referências Bibliográficas}

ALMEIDA FILHO, N.; ROUQUAYROL, M. Z. Introdução à epidemiologia moderna. 2ed. Rio de Janeiro: Coopemed/APCE/ Abrasco, 1992.

CARNEIRO, C. et al. Influência da distribuição da gordura corporal sobre a prevalência de hipertensão arterial e outros fatores de risco cardiovascular em indivíduos obesos. Rev. Assoc. Med. Bras., v. 49, n. 3, 2003.

COSTA, M. C. Práticas Alimentares, sobrepeso e perfil lipídico dos trabalhadores de uma indústria petroquímica, CamaçariBahia. 2000. Dissertação (Mestrado em Nutrição) - Programa de Pós-Graduação da Escola de Nutrição da Universidade Federal da Bahia, Salvador, 2000.

EPSTEIN, F. H.; HIGGINS, M. Epidemiology of obesity. In: BJÖRNTORP, P; BRODOFF, B. N. Obesity. Philadelphia: J. B. Lippincott Company, v. 1, p. 330-342, 1992.

FLEGAL, M. D. et al. Overweight and obesity in the United States: prevalence and trends, 1960-1994. Int. J. Obes. Relat. Metab. Disord., n. 22, p. 39-47, 1998.

FONSECA, M. H. G. A. et al. Obesidade. Ars Cvrandi, v. 32, n. 8, p. 24-36, 1999.

HAN, T. S. et al. Waist circunference action levels in the identification of cardio- problemas a ele associados, como as dislipidemias e a adiposidade central, fatores de risco para o desenvolvimento de doenças crônicas não-transmissíveis - DCNT.

Reconhecendo no CESAT um centro de referência estadual para a abordagem da saúde do trabalhador, os resultados respaldam a importância de se produzir dados que permitam conhecer a realidade da saúde da população atendida, buscando articular ações de assistência ao trabalhador com aquelas de prevenção de agravos e de controle de seus determinantes. Uma política institucional orientada pela prática sanitária de vigilância à saúde voltada para a gestão dos riscos populacionais recupera a idéia de que tais práticas, sobretudo as de prevenção de riscos e promoção à saúde, possam e devam desenvolver-se nos vários espaços, considerando a complexidade dos problemas estudados e o ônus que eles representam para o Estado e para o conjunto da população economicamente ativa.

vascular risk factors: prevalence study in a random sample. Br. Med. J. v. 25, n. 7017, p. 1401-1405, 1995.

HALPERN, A; MANCINI, M. C. MedicaÇões contra obesidade. Quais? Quando? Como? Até quando? Rev. Bras. Nutr. Clin., v. 15, p. 384-390, 2000.

HUBERT, H. B. et al. Obesity as an independent risk factor for cardiovascular disease: a 26 year follow-up of participants in the Framingham Heart Study. Circulation, v. 67, n.5, p. 968-977, 1983.

KAC, G. et al. Fatores associados à obesidade abdominal em mulheres em idade reprodutiva. Rev. Saúde Pública, v. 35, n. 1, p. 46-51, 2001.

LESSA, I. Doenças crônicas não-transmissíveis. In:___. (org.) O adulto brasileiro e as doenças da modernidade: epidemiologia das doenças crônicas não-transmissíveis. São Paulo: Hucitec, 1998.

LESSA, I. et al. Obesidade central: associação com outros fatores de risco cardiovascular e para o diabetes. In: CONGRESSO BRASILEIRO DE EPIDEMIOLOGIA - EPI 2004, 6, 2004, Recife, Pôster. Recife, 2004.

MARTINEZ, T. L. R. Condutas clínicas nas dislipidemias. Belo Horizonte: Saúde, 1997. 
MONDINI, L.; MONTEIRO, C. A. Mudanças no padrão de alimentação. In: MONTEIRO, C. A. (org.) Velhos e novos males da saúde no Brasil: a evolução do país e de suas doenças. São Paulo: Hucitec, 1995. p. 7989.

MONTEIRO, C. A. et al. Da desnutrição para a obesidade: a transição nutricional no Brasil. In: MONTEIRO, C. A. (org.) Velhos e novos males da saúde no Brasil: a evolução do país e de suas doenças. São Paulo: Hucitec, 1995. p. 247-255.

MONTEIRO, C. A.; CONDE, W. L. A tendência secular da obesidade segundo estratos sociais: Nordeste e Sudeste do Brasil. Arq. Bras. Endocrinol. Metab., v. 43, n. 3, p. 186-194, 1999.

SOCIEDADE BRASILEIRA DE CARDIOLOGIA (SBC). Diretrizes brasileiras e diretriz de prevenção da aterosclerose do Departamento de Aterosclerose da Sociedade Brasileira de Cardiologia. Arq. Bras. Cardiol., v. 77, supl. III, 2001.

. Diretrizes Brasileiras de Hipertensão Arterial. Arq. Bras. Cardiol., v. 82, supl. IV, 2004.

SOCIEDADE DE CARDIOLOGIA DO ESTADO DE SÃO PAULO (SOCESP). Dislipidemias e aterosclerose. Revista SOCESP. v. 10, n. 6, 2000.
SOCIEDADE BRASILEIRA DE HIPERTENSÃO (SBH); SOCIEDADE BRASILEIRA DE CARDIOLOGIA (SBC); SOCIEDADE BRASILEIRA DE NEFROLOGIA (SBN). Diretrizes Brasileiras de Hipertensão Arterial. Arq. Bras. Cardiol., v. 82, supl. IV, 2004.

SUPLICY, H. L. Obesidade visceral, resistência à insulina e hipertensão arterial. Rev. Bras. Hipertens., v. 7, n. 2, p. 136-141, 2000.

VELOSO, I. S.; SANTANA, V. Impacto nutricional do programa de alimentação do trabalhador no Brasil. Rev. Panam. Salud Públ., v.11, n.1, p. 24-31, 2002.

VIANA, S. V. Indústria moderna e padrão alimentar: o espaço do trabalho, do consumo e da saúde. 2000. Tese (Doutorado em Saúde Pública) - Programa de Pós-Graduação em Saúde Coletiva, Universidade Federal da Bahia, Salvador, 2000.

WORLD HEALTH ORGANIZATION (WHO). Dieta, nutrición y prevención de enfermedades crónicas: informe de una consulta mixta FAO/OMS de expertos (OMS, Serie de Informes Técnicos, 916). Ginebra, 2003.

Obesity: preventing and managing the global epidemic - Report of a WHO consultation on obesity (Programme of Nutrition Family and Reproductive Health). Geneva, 1997. 